PharmacoEconomics \& Outcomes News 880, p10 - 12 Jun 2021

\title{
Economic impact of avoidable cancer deaths due to COVID-19 pandemic 'substantial'
}

A study published in the European Cancer Journal examining the economic impact of avoidable cancer deaths from delays in diagnosis resulting from the first wave of the COVID-19 pandemic in England has shown substantial health and economic losses. ${ }^{1}$

The researchers used the human capital approach to model the health losses (in quality-adjusted life years [QALYs]) and lost productivity in England. Data were linked from the NHS National Health Service cancer registration and hospital administrative datasets for patients aged 15-84 years, diagnosed with either breast, colorectal, oesophageal or lung cancer with suitable follow-up data. Productivity losses were based on excess additional deaths from cancer at years 1,3 and 5 derived from a previous dataset. Five hundred random samples were taken from national statistics to compare productivity losses resulting from COVID-19-related deaths. In total, there were 32583 patients with breast cancer, 24975 with colorectal cancer, 6744 with oesophageal cancer, and 29305 with lung cancer. Across these four cancer types only in England, additional excess cancer deaths would equate to a loss of 32700 QALYs and productivity losses of $£ 103.8$ million over the next 5 years. Respective, QALY and productivity losses according to cancer type were 4100 QALYs and $£ 23.2$ million for breast cancer, 15000 QALYs and $£ 35.7$ million for colorectal cancer, 10 900 QALYs and $£ 38.3$ million for lung cancer, and, 2700 QALYs and £6.6 million for oesophageal cancer. The equivalent number of COVID-19 deaths caused approximately 21450 QALYs lost and productivity losses of $£ 76.4$ million.

\section{A 'lasting legacy' for all nations}

In an accompanying editorial, Pat Price of the Imperial College London (London, England) says the "timely, wellsourced analysis" demonstrates the lasting health and economic burden that nations will face in their journey to recover from the impact of the COVID-19 pandemic. $^{2}$

Price highlights that the per capita losses in QALYs and costs due to excess cancer deaths over 5 years are nearly 1.5 times higher than those related to COVID-19, and that this is a conservative estimate as it does not include additional productivity losses from delays or reduction in quality of treatment and stage migration. But more importantly, Price says this analysis does not include all cancer types across all COVID-19 waves in all nations in Europe - the "impact of excess cancer deaths due to COVID-19-induced diagnostic and treatment delays in Europe is going to be catastrophic" and the effects of the backlog in delayed diagnosis and treatment will last for many years.

The effects of public health policy to manage the COVID-19 pandemic suggest the leadership lacked the welfare and economic input to balance the wider consequences of public health actions, says Price, "services need to learn from the major disruption caused by the pandemic and be made fit for purpose for the future".

1. Gheorghe A, et al. Economic impact of avoidable cancer deaths caused by diagnostic delay during the COVID-19 pandemic: A national population-based modelling study in England, UK. European Journal of Cancer : 5 May 2021. Available from: URL: http://doi.org/10.1016/j.ejca.2021.04.019.

2. Price P. Cancer during the COVID-19 pandemic: did we shout loudly enough and did anyone listen? A lasting legacy for nations. European Journal of Cancer : 1 Jun 2021. Available from: URL: https://doi.org/10.1016/j.ejca.2021.04.020. 